\title{
Cobalt induces oxidative stress in isolated liver mitochondria responsible for permeability transition and intrinsic apoptosis in hepatocyte primary cultures
}

\author{
Valentina Battaglia $^{\mathrm{a}, 1}$, Alessandra Compagnone ${ }^{\mathrm{b}, 1}$, Andrea Bandino $^{\mathrm{b}}$, \\ Marcantonio Bragadin ${ }^{\mathrm{c}}$, Carlo Alberto Rossi ${ }^{\mathrm{a}}$, Filippo Zanetti ${ }^{\mathrm{a}}$, \\ Sebastiano Colombatto ${ }^{\mathrm{b}}$, Maria Angelica Grillo ${ }^{\mathrm{b}}$, Antonio Toninello ${ }^{\mathrm{a}, *}$ \\ a Dipartimento di Chimica Biologica, Università degli Studi di Padova, Istituto di Neuroscienze del CNR, Padova, Italy \\ ${ }^{\mathrm{b}}$ Dipartimento di Medicina e Oncologia Sperimentale, Sezione di Biochimica, Università di Torino, Torino, Italy \\ ' Dipartimento di Scienze Ambientali, Università Ca' Foscari, Venezia, Italy
}

\section{A R T I C L E I N F O}

\section{Article history:}

Received 22 January 2008

Received in revised form 16 July 2008

Accepted 16 July 2008

Available online 31 July 2008

\section{Keywords:}

Isolated liver mitochondria

Hepatocytes primary cultures

Cobalt

Oxidative stress

Apoptosis

\begin{abstract}
A B S T R A C T
It is well established that cobalt mediates the occurrence of oxidative stress which contributes to cell toxicity and death. However, the mechanisms of these effects are not fully understood. This investigation aimed at establishing if cobalt acts as an inducer of mitochondrial-mediated apoptosis and at clarifying the mechanism of this process.

Cobalt, in the ionized species $\mathrm{Co}^{2+}$, is able to induce the phenomenon of mitochondrial permeability transition (MPT) in rat liver mitochondria (RLM) with the opening of the transition pore. In fact, $\mathrm{Co}^{2+}$ induces mitochondrial swelling, which is prevented by cyclosporin A and other typical MPT inhibitors such as $\mathrm{Ca}^{2+}$ transport inhibitors and bongkrekic acid, as well as anti-oxidant agents. In parallel with mitochondrial swelling, $\mathrm{Co}^{2+}$ also induces the collapse of electrical membrane potential. However in this case, cyclosporine A and the other MPT inhibitors (except ruthenium red and EGTA) only partially prevent $\Delta \Psi$ drop, suggesting that $\mathrm{Co}^{2+}$ also has a proton leakage effect on the inner mitochondrial membrane. MPT induction is due to oxidative stress, as a result of generation by $\mathrm{Co}^{2+}$ of the highly damaging hydroxyl radical, with the oxidation of sulfhydryl groups, glutathione and pyridine nucleotides. $\mathrm{Co}^{2+}$ also induces the release of the pro-apoptotic factors, cytochrome $c$ and AIF. Incubation of rat hepatocyte primary cultures with $\mathrm{Co}^{2+}$ results in apoptosis induction with caspase activation and increased level of expression of HIF- $1 \alpha$.

All these observations allow us to state that, in the presence of calcium, $\mathrm{Co}^{2+}$ is an inducer of apoptosis triggered by mitochondrial oxidative stress.
\end{abstract}

(C) 2008 Elsevier Ltd. All rights reserved.

\section{Introduction}

Cobalt is an oligoelement present in almost all the animal and vegetal organisms; its biological importance is due to its essen-

Abbreviations: AdNT, adenine nucleotide translocase; AIF, apoptosis inducing factor; APF, 2-[6-(4'-amino)phenoxy-3H-xanthen-3-on-9-yl]benzoic acid; BHT, butylhydroxytoluene; BKA, bongkrekic acid; DMF, dimethyl formamide; CsA, cyclosporin A; cyt $c$, cytochrome $c$; DTE, dithioerythritol; HIF-1, hypoxia-inducible factor-1; MPT, mitochondrial permeability transition; NAC, $N$-acetylcysteine; RLM, rat liver mitochondria; ROS, reactive oxygen species; RR, ruthenium red; TBARS, thiobarbituric acid-reactive species; $\Delta \Psi$, membrane potential.

* Corresponding author at: Dipartimento di Chimica Biologica, Università degli Studi di Padova, Istituto di Neuroscienze del C.N.R., Unità per lo Studio delle Biomembrane, Viale G. Colombo 3, 35121 Padova, Italy. Tel.: +39 0498276134; fax: +390498276133 .

E-mail address: antonio.toninello@unipd.it (A. Toninello).

1 These authors contributed equally to this work. tial role in the formation of vitamin $\mathrm{B}_{12}$ and other cobalamines. Vitamin $B_{12}$ is necessary for the organism, because it is involved in the formation of some proteins and in the normal functionality of the nervous system. Its lack can cause pernicious anaemia and peripheral nervous system diseases (Karovic et al., 2006).

Cobalt is potentially toxic in the ionic form, $\mathrm{Co}^{2+}$. Data in the literature indicate that cobalt is cytotoxic to many cell types, including neural cells (Wang et al., 2000) and can induce cell death by apoptosis and necrosis (Huk et al., 2004). It can cause DNA fragmentation (Zou et al., 2001), activation of caspases (Zou et al., 2002), increased production of reactive oxygen species (ROS) (Olivieri et al., 2001), augmented phosphorylation of mitogen-activated protein (MAP) kinases (Yang et al., 2004), and elevated levels of p53 (Chandel et al., 2000), as a consequence of the activation of hypoxia-inducible factor-1 (HIF-1) (Zou et al., 2001). In fact, in cultured cells, cobalt chloride mimics a hypoxic response. Like low oxygen tension, this metal is able to stabilize the $\alpha$-subunit of HIF-1 (HIF- $1 \alpha$ ) by block- 
ing its ubiquitination and proteasomal degradation (Epstein et al., 2001; Morwenna and Ratcliffe, 1997). Increased levels of HIF-1 $\alpha$ stimulate overexpression of a set of genes encoding several proteins such as heat shock proteins, which promote a physiological response linked to the recovery of cell homeostasis. In the same way the transcription of many pro-apoptotic factors, such as NIP-3 and NIX, is achieved, with the effect of leading to cell death (Bruick, 2000).

Many experiments have been performed on alveolar macrophages and PC12 cells (Zou et al., 2001; Tomaro et al., 1991). The way by which $\mathrm{Co}^{2+}$ is able to induce apoptosis still has to be discovered, but there is some evidence that it activates both the extrinsic and the intrinsic pathway. Zou et al. used a caspase 3-like inhibitor, which is able to inhibit programmed cell death partially, suggesting the peculiar role of this protein in the cobalt-mediated process (Zou et al., 2002). In spite of these observations, the molecular mechanism by means of which cobalt leads to cell death still has to be understood.

There is some evidence that it acts by activating the intrinsic apoptotic pathway, because its effect is blocked by caspase 9-inhibitors (Araya et al., 2002). This suggests that production of ROS induced by the metal acts directly on mitochondria to provoke the release of cytochrome $c$ (cyt $c$ ) from external mitochondrial membrane, which leads to the activation of caspase 9 and to apoptosis (Pulido and Parrish, 2003). Similar conclusions have also been reported by other authors studying the toxic effects of cobalt in primary cultures of mouse astrocytes (Karovic et al., 2006). The interaction of $\mathrm{Co}^{2+}$ with mitochondrial function has been preliminarily investigated at the level of ATP synthesis, with inhibition of this phenomenon, probably ascribable to the opening of the transition pore (Bragadin et al., 2007).

The aim of our work is to explain the mechanism of cobaltinduced cell death and which is the role of mitochondria in this phenomenon. Our studies were performed on hepatocyte primary cultures and isolated liver mitochondria, because the highest quantities of physiological $\mathrm{Co}^{2+}$ in the body is contained in the liver, as in kidney, heart and spleen, whereas low concentrations are detected in serum, brain and pancreas (Derelank and Hollinger, 2002).

\section{Materials and methods}

\subsection{Materials}

Mouse monoclonal antibody anti-cyt $c$ was purchased from Pharmingen, rabbit polyclonal antibody anti-apoptosis-inducing factor (AIF) was purchased from Chemicon International. Rabbit polyclonal antibody anti-caspase 3 and rabbit polyclonal antibody anti-HIF- $1 \alpha$ were purchased from Santa Cruz Biothecnology. Fluorescence probe 2-[6-(4'-amino)phenoxy-3H-xanthen-3-on-9yl]benzoic acid (APF) was from Sigma. All other reagents were of the highest purity commercially available.

\subsection{Mitochondrial isolation and standard incubation procedures}

Rat liver mitochondria (RLM) isolated by conventional differential centrifugation in a buffer containing $250 \mathrm{mM}$ sucrose, $5 \mathrm{mM}$ HEPES (pH 7.4), and 1 mM EGTA (Schneider and Hogeboom, 1950); EGTA was omitted from the final washing solution. Protein content was measured by the biuret method with bovine serum albumin as a standard (Gornall et al., 1949). Mitochondria (1 mg protein/ml) incubated in a water-jacketed cell at $20^{\circ} \mathrm{C}$. The standard medium contained $250 \mathrm{mM}$ sucrose, $10 \mathrm{mM}$ HEPES (pH 7.4), $5 \mathrm{mM}$ succinate, $50 \mu \mathrm{M} \mathrm{Ca}^{2+}$, and $1.25 \mu \mathrm{M}$ rotenone. Variations and/or other additions are given with each experiment.
The experiments were carried out at $20^{\circ} \mathrm{C}$ in order to compare the results with those obtained in many other previous papers on the mitochondrial permeability transition (MPT) (e.g., see Gardini et al., 2001; DallaVia et al., 2006). Whole rat liver mitochondria exhibit a reversible broad gel to liquid crystalline phase transition at $0{ }^{\circ} \mathrm{C}$ (Blazyk and Steim, 1972) and at $20^{\circ} \mathrm{C}$ the membrane is in the sol form. In MPT conditions, the fluidity of the membrane is greatly increased with respect to control conditions and increases still further as temperatures rise (Ricchelli et al., 1999). Therefore, the choice of $20^{\circ} \mathrm{C}$ was made with the aim of minimizing alteration of the membrane during the MPT due to excessive fluidity. It should also be emphasized that, at higher temperatures, e.g., $30^{\circ} \mathrm{C}$, the respiratory chain operates at a high rate, producing anaerobiosis in the mitochondrial suspension within a few minutes, particularly in MPT conditions.

\subsection{Determination of mitochondrial functions}

Membrane potential $(\Delta \Psi)$ was calculated on the basis of movement of the lipid-soluble cation tetraphenylphosphonium $\left(\mathrm{TPP}^{+}\right)$through the inner membrane, measured using a $\mathrm{TPP}^{+}$-specific electrode (Kamo et al., 1979). $\Delta \Psi$ determinations were corrected for non-specific intramitochondrial binding of $\mathrm{TPP}^{+}$, as proposed by Jensen et al. (1986). Mitochondrial swelling was determined by measuring the apparent absorbance change of mitochondrial suspensions at $540 \mathrm{~nm}$ in a Kontron Uvikon model 922 spectrophotometer equipped with thermostatic control.

The protein sulfhydryl group oxidation assay was performed as in Santos et al. (1998). The redox level of glutathione was monitored as described in Tietze (1969). The redox state of endogenous pyridine nucleotides was followed fluorometrically in an AmincoBowman 4-8202 spectrofluorometer with excitation at $354 \mathrm{~nm}$ and emission at $462 \mathrm{~nm}$.

The production of $\mathrm{H}_{2} \mathrm{O}_{2}$ in mitochondria was measured fluorometrically by the Scopoletin method (Loschen et al., 1973) in an Aminco-Bowman 4-8202 spectrofluorometer.

Hydroxyl radical was detected fluorometrically by the probe APF with excitation at $490 \mathrm{~nm}$ and emission at $555 \mathrm{~nm}$ according to Setsukinai et al. (2003).

Lipid peroxidation was determined by monitoring the formation of thiobarbituric acid-reactive species (TBARS) according to Willis and Wilkinson (1966). TBARS were determined spectrofluorimetrically at $532 \mathrm{~nm}$ with an extinction coefficient of $1.56 \times 10^{5} \mathrm{M}^{-1} \mathrm{~cm}^{-1}$. Protein carbonyls were measured spectrophotometrically at $360 \mathrm{~nm}$ with the extinction coefficient of $22,000 \mathrm{M}^{-1} \mathrm{~cm}^{-1}$, according to Reznick and Packer (1994).

The cobalt ion content of the supernatant and its fluxes across the membrane were estimated by a centrifugal-filtration method (Toninello et al., 1985) with atomic absorption spectroscopy, on a Perkin-Elmer 110B spectrometer.

\subsection{Detection of cyt $c$ and AIF release}

Mitochondria ( $1 \mathrm{mg}$ protein $/ \mathrm{ml}$ ) were incubated for $15 \mathrm{~min}$ at $20^{\circ} \mathrm{C}$ in standard medium with the appropriate additions. The reaction mixtures were then centrifuged at $13,000 \times \mathrm{g}$ for $10 \mathrm{~min}$ at $4{ }^{\circ} \mathrm{C}$ to obtain mitochondrial pellets. The supernatant fractions were further spun at $100,000 \times g$ for $15 \mathrm{~min}$ at $4{ }^{\circ} \mathrm{C}$ to eliminate mitochondrial membrane fragments and concentrated five times by ultrafiltration through Centrikon 10 membranes (Amicon) at $4{ }^{\circ} \mathrm{C}$. Aliquots of $10 \mu \mathrm{l}$ of the concentrated supernatants were subjected to $15 \%$ SDS-PAGE for cyt $c$ and $10 \%$ SDS-PAGE for AIF and analyzed by western blotting using mouse anti-cyt $c$ antibody and rabbit anti-AIF antibody. 


\subsection{Rat hepatocytes preparation and culture}

Male Wistar rats weighing 150-200g were used to isolate hepatocytes by the collagenase perfusion method (Probst and Unthan-Fechner, 1982). Their care was in accordance with the national guidelines for animal experimentation. The cells were plated at a density of $7 \times 10^{4}$ viable cells $/ \mathrm{cm}^{2}$ on culture dishes coated with rat tail tendon collagen in M199 medium supplemented with $2 \mathrm{mg}$ bovine serum albumin, $3.6 \mathrm{mg}$ Hepes, $100 \mathrm{U}$ penicillin, $100 \mu \mathrm{g}$ streptomycin/ml, $5 \%$ horse serum and $1 \mathrm{nmol} / \mathrm{l}$ insulin, and incubated in a humidified incubator with a $\mathrm{CO}_{2} / \mathrm{air}$ atmosphere $(5: 95, \mathrm{v} / \mathrm{v})$. Only cell suspensions with a vitality (tested by trypan blue exclusion) of $70 \%$ or more were used. After $4 \mathrm{~h}$ for cell attachment, the medium was changed to M199 as above, but without horse serum with $10 \mathrm{nmol} / \mathrm{l}$ insulin, and incubated in a $\mathrm{CO}_{2}$ /air atmosphere $(5: 95, \mathrm{v} / \mathrm{v})$ atmosphere. The medium was changed daily.

\subsection{Morphological evaluation of apoptosis}

Rat hepatocytes were cultured for $48 \mathrm{~h}$. After washing and fixation the cells were incubated with Hoechst dye $33258(8 \mu \mathrm{g} / \mathrm{ml})$ in PBS $\left(0.8 \% \mathrm{NaCl}, 0.02 \% \mathrm{KCl}, 0.115 \% \mathrm{Na}_{2} \mathrm{HPO}_{4}\right.$ and $0.02 \% \mathrm{KH}_{2} \mathrm{PO}_{4}$, $\mathrm{pH}$ 7.2) for $5 \mathrm{~min}$, washed with PBS and mounted with glycerol in phosphate buffered saline. For each experimental observation the percentage of apoptotic cells per 1000 cells was scored by fluorescent microscopy (Leica DMLB).

\subsection{Caspase-3 activation and HIF-1 $\alpha$ accumulation}

At the end of the culture, hepatocytes were washed twice with PBS and collected with boiling loading buffer. After sonication $30 \mu \mathrm{g}$ protein were analyzed by SDS-PAGE on a polyacrylamide gel $(15 \%$ for caspase 3 and $7.5 \%$ for HIF-1 $\alpha$ ). Standard protein markers were used for molecular weight calibration. After blotting, immunoreaction bands were detected by ECL (Gardini et al., 2001).

\subsection{Statistical analysis}

One-way analysis of variance (ANOVA) was applied to the data of Fig. 4. Statistical analysis was performed with SPSS 10.0 (Norusis, 1993). All probabilities are two-tailed. Data were cheked for normality and homogeneity of variance (Leven test). Differences between means were evaluated for significance by using Duncan's multiple range test (DMRT) $(p<0.05)$.

\section{Results}

In a previous paper, it is reported that, over the concentration range of $0.2-0.8 \mathrm{mM}, \mathrm{Co}^{2+}$ has toxic effects on primary cultures of mouse astrocytes (Karovic et al., 2006). These cobalt levels are much higher than those of plasma measured during human exposure to $\mathrm{Co}^{2+}$ but almost identical to the concentrations used in other investigations of metabolism (Karovic et al., 2006). However, in a previous study we had demonstrated that, at lower concentrations - e.g., 5-15 $\mu \mathrm{m}-\mathrm{Co}^{2+}$ inhibits ATP synthesis in a dose-dependent manner (Bragadin et al., 2007). Taking these observations into account, we used $\mathrm{Co}^{2+}$ at a concentration of $10 \mu \mathrm{M}$ in isolated RLM and $200 \mu \mathrm{M}$ in hepatocyte cultures.

The results reported in Fig. 1 show that energized RLM, incubated in standard medium, in the presence of a supraphysiological $\mathrm{Ca}^{2+}$ concentration $(50 \mu \mathrm{M})$, when treated with $\mathrm{Co}^{2+}$ exhibit a dose-dependent decrease of the apparent absorbance of their suspension, with a maximum of about 1 unit, indicative of the

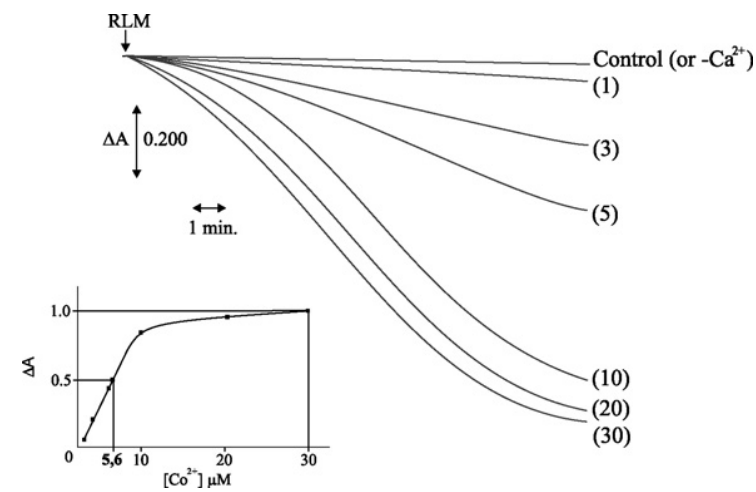

Fig. 1. Dose-dependent induction of mitochondrial swelling by cobalt. RLM were incubated for $15 \mathrm{~min}$ in standard medium as described in Section 2. Control refers to the experiment with $\mathrm{Ca}^{2+}$. $\mathrm{Co}^{2+}$ was present at $\mu \mathrm{M}$ concentrations indicated on side of curves. Inset: calculation of $S_{0.5}$ value of $\mathrm{Co}^{2+}$. Experiment replicated seven times, with comparable results.

occurrence of large amplitude matrix swelling. The $\mathrm{Co}^{2+}$ concentration able to induce the half maximum absorbance decrease $\left(S_{0.5}\right)$ is $5.6 \mu \mathrm{M}$ (Fig. 1, inset) which is in agreement with our choice to use $10 \mu \mathrm{M} \mathrm{Co}^{2+}$ in RLM. Fig. 2 shows the effects of different wellknown inhibitors of MPT on the swelling induced by $10 \mu \mathrm{M} \mathrm{Co}^{2+}$ in the presence of $\mathrm{Ca}^{2+}$. As Fig. 2 shows, this swelling is completely prevented by the immunosuppressant cyclosporin $\mathrm{A}$ (CsA) and by the inhibitors of $\mathrm{Ca}^{2+}$ transport, ruthenium red (RR) and EGTA (panel A), the adenine nucleotide translocase (AdNT) ligands, ADP, ATP and bongkrekic acid (BKA) (panel B) and the reductants dithioerythritol (DTE) and $N$-acetylcysteine (NAC) and the anti-oxidant butylhydroxytoluene (BHT) (panel C). Instead, the divalent cations $\mathrm{Mg}^{2+}$ and $\mathrm{Mn}^{2+}$ are weak inhibitors (panel D). It is noteworthy that $\mathrm{Ca}^{2+}$ alone (see control $+\mathrm{Ca}^{2+}$ in panel $\mathrm{A}$ ) and $\mathrm{Co}^{2+}$ alone (panel $\mathrm{B}$ ) are completely ineffective. In parallel with osmotic alterations, $\mathrm{Co}^{2+}$, again in the presence of $\mathrm{Ca}^{2+}$, induces the collapse of $\Delta \Psi$ (Fig. 3, all panels). In this case the above mentioned inhibitors, except RR and EGTA (panel A), only partially prevent $\Delta \Psi$ collapse (panels B and C), whereas the divalent cations, also in this case, fail to show protection (panel D). It should be emphasized that $\mathrm{Ca}^{2+}$ alone, and $\mathrm{Co}^{2+}$ in the absence of $\mathrm{Ca}^{2+}$, are completely ineffective in inducing $\Delta \Psi$ collapse (Fig. 3, panels A and D). However, higher concentrations of $\mathrm{Co}^{2+}(200 \mu \mathrm{M})$ do cause a $\Delta \Psi$ drop, which cannot be prevented by CsA or the other agents, suggesting aspecific membrane damage (Fig. 8, inset). The observation that DTE, NAC and BHT almost completely inhibit mitochondrial swelling induced by $\mathrm{Co}^{2+}$ suggests that these events are linked to oxidative stress. The results shown in the subsequent figures solve this question. As shown in the histogram of Fig. $4 \mathrm{~A}, 10 \mu \mathrm{M} \mathrm{Co}^{2+}$ in the presence of $50 \mu \mathrm{M} \mathrm{Ca}^{2+}$ induces a decrease in the content of reduced sulfhydryl groups by about $40 \%$. Note that $\mathrm{Ca}^{2+}$ and $\mathrm{Co}^{2+}$ alone, at the above concentrations, induce a very low but statistically significative oxidation of thiols, of about 2 and $4.5 \%$, respectively (see columns control and $\mathrm{Co}^{2+}-\mathrm{Ca}^{2+}$ in Fig. 4A). Almost similar effects by $\mathrm{Ca}^{2+}$ and $\mathrm{Co}^{2+}$ are observable on glutathione oxidation (Fig. 4B). Fig. 5 shows that $\mathrm{Co}^{2+}$, in the presence of $\mathrm{Ca}^{2+}$, induces a considerable decrease in fluorescence of the $\mathrm{NAD}(\mathrm{P}) \mathrm{H}$ pool present in mitochondria. This event, which is concomitant with the decrease in apparent absorbance due to MPT induction, is indicative of considerable oxidation of pyridine nucleotides. This oxidation is strongly attenuated in the absence of $\mathrm{Ca}^{2+}$. Also in this case $\mathrm{Ca}^{2+}$ alone (control) induces a negligible oxidation. The pro-oxidant effect demonstrated by $\mathrm{Co}^{2+}$ in these experiments suggests the possibility that this ion is responsible for the production of some ROS. The results of Fig. 6 demonstrate that $\mathrm{Co}^{2+}$ alone induces an increase of $\mathrm{H}_{2} \mathrm{O}_{2}$ production as well as $\mathrm{Ca}^{2+}$ 

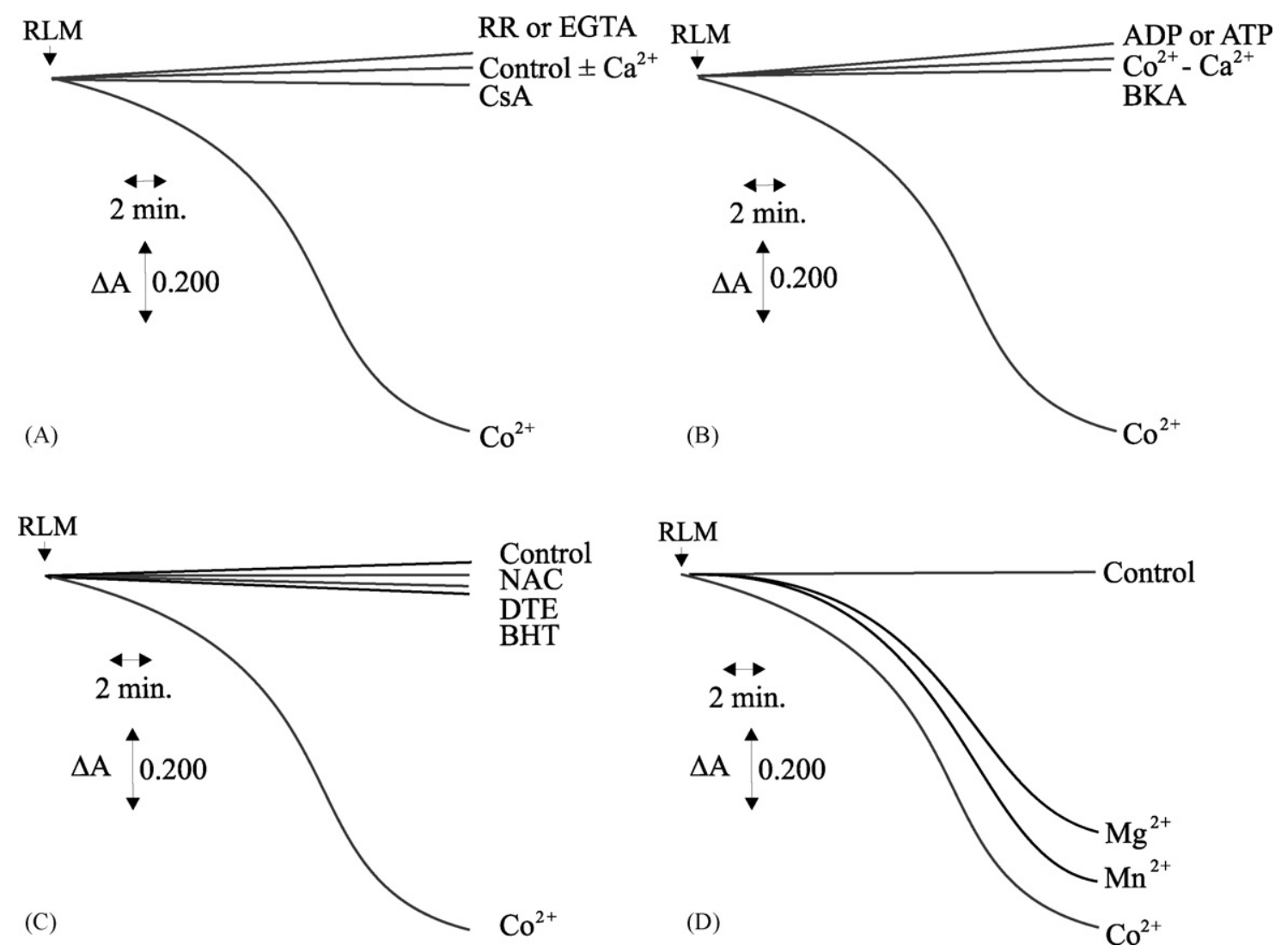

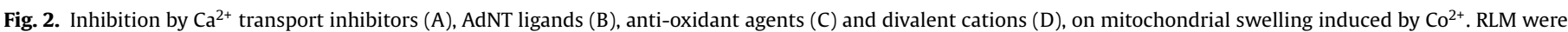

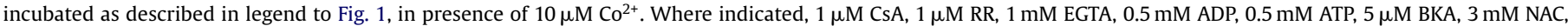
$1 \mathrm{mM}$ DTE, $25 \mu \mathrm{M}$ BHT, $1 \mathrm{mM} \mathrm{Mg}^{2+}$ and $1 \mathrm{mM} \mathrm{Mn}^{2+}$ were present in medium. Experiment replicated four times with almost identical results.

(control), if compared with the curve without $\mathrm{Ca}^{2+}$, the presence of $\mathrm{Ca}^{2+}$ the production of this ROS by $\mathrm{Co}^{2+}$ is further increased. The increase in the oxidations and $\mathrm{H}_{2} \mathrm{O}_{2}$ production are strongly prevented by CsA (Figs. 4-6A). In order to obtain a further specific indication on the ROS responsible of the observed oxidations, RLM were pre-treated for $1 \mathrm{~min}$ with the fluorescence probe APF and subsequently undergone to the action of $\mathrm{Co}^{2+}$ or $\mathrm{Ca}^{2+}$ alone
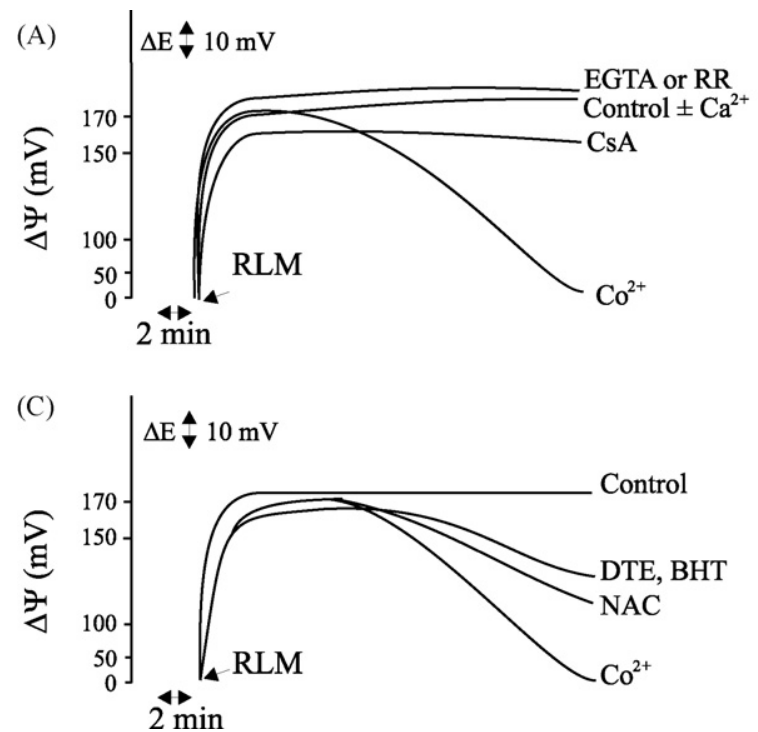

or together. The results of Fig. 6B show that $\mathrm{Co}^{2+}$ is also able to induce, as well as $\mathrm{Ca}^{2+}$, an increase in the fluorescence of mitochondrial suspension, indicative of highly ROS generation. Addition of both the cations together induces a further increase of these ROS.

Indeed, the results of Fig. 7 show that, besides an increase in $\mathrm{H}_{2} \mathrm{O}_{2}$ and highly ROS generation, $\mathrm{Co}^{2+}$ alone can induce a consis-
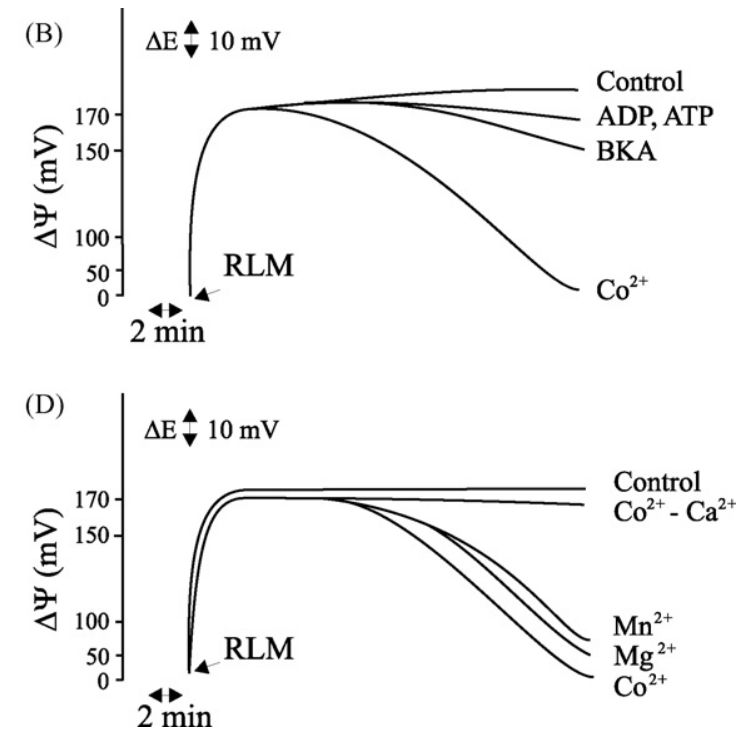

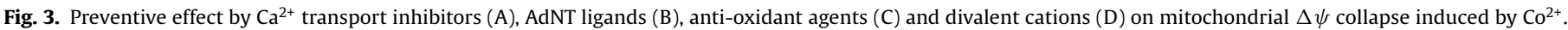
Incubation conditions and compound concentrations as in Fig. 2. Experiment replicated four times, with comparable results. 

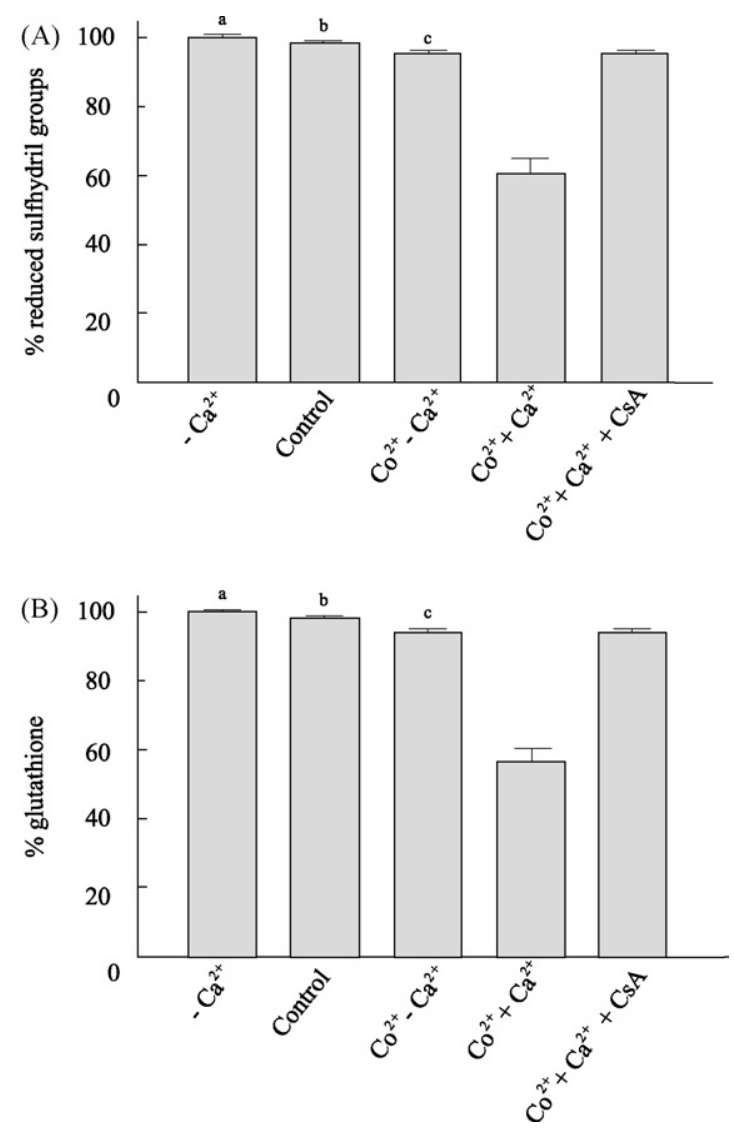

Fig. 4. Changes in redox level of mitochondrial thiols and glutathione induced by $\mathrm{Co}^{2+}$. RLM were incubated for $15 \mathrm{~min}$ in standard medium, as described in Section 2. Where indicated $\left(-\mathrm{Ca}^{2+}\right)$, medium was deprived of $\mathrm{Ca}^{2+} \cdot \mathrm{Co}^{2+}$ was present at $10 \mu \mathrm{M}$ concentration. When present CsA was $1 \mu \mathrm{M}$. Data are expressed as percentage of thiol or glutathione reduction, and represent average \pm mean S.D. of six independent experiments. Values followed by different letters are significantly different $(p<0.05)$, as determined by DMTR.

tent increase in TBARS production of about $40 \%$, indicative of lipid peroxidation.

An attempt was also made to evaluate if $\mathrm{Co}^{2+}$ can oxidize lateral aminoacid residues of mitochondrial membrane proteins, but results were negative (results not reported).

All these observations suggest that $\mathrm{Co}^{2+}$ is transported into the inner compartment of RLM. The experiment (Fig. 8) was performed in order to confirm this possibility. Results show that $\mathrm{Co}^{2+}$,

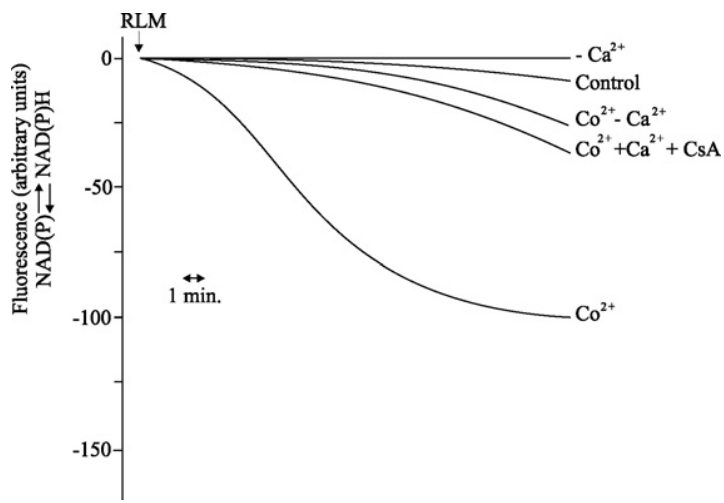

Fig. 5. Changes in redox level of mitochondrial pyridine nucleotides by $\mathrm{Co}^{2+}$. Incubation conditions and compounds concentrations as in Fig. 4. Experiments replicated three times gave very similar results.
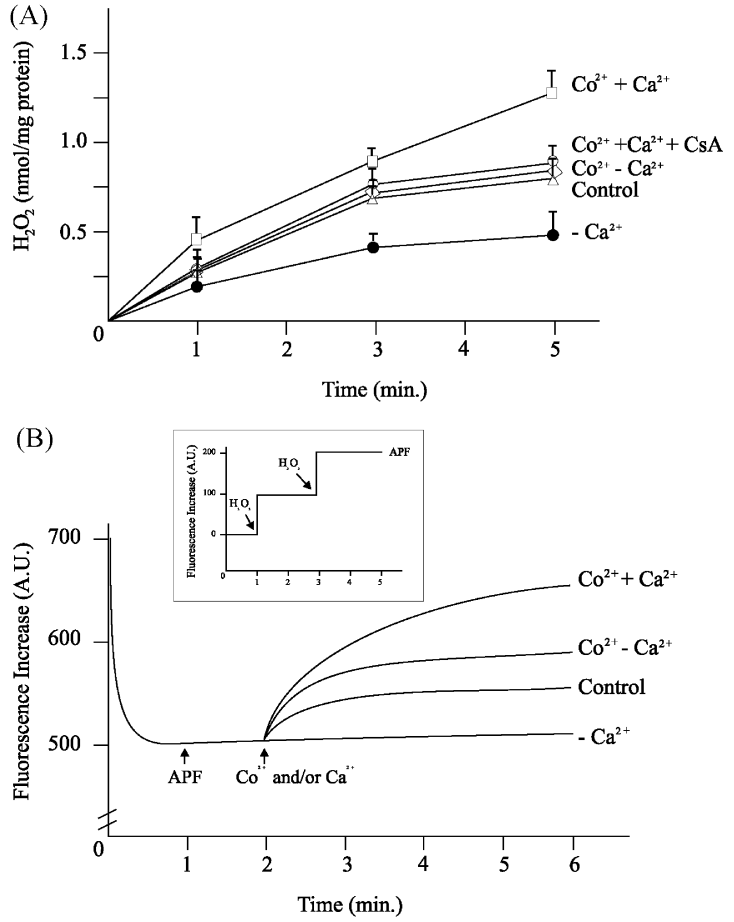

Fig. 6. Increased generation of hydrogen peroxide by RLM induced by $\mathrm{Co}^{2+}(\mathrm{A})$ and detection of hydroxyl radical (B). (A) Incubation conditions and compounds concentration as in Fig. 4. Mean values \pm S.D. of four experiments. (B) $R L M(0.5 \mathrm{mg} / \mathrm{ml})$ were incubated in standard medium deprived of $\mathrm{Ca}^{2+}$, in the conditions described in Section 2. $10 \mu \mathrm{M}$ APF (solved in $0.1 \%$ DMF) was added, as indicated, $1 \mathrm{~min}$ before $\mathrm{Co}^{2+}$ and $\mathrm{Ca}^{2+}$, which were 10 and $50 \mu \mathrm{M}$, respectively. The inset shows the detection of hydroxyl radical produced by two subsequent additions of $1 \mathrm{mM} \mathrm{H}_{2} \mathrm{O}_{2}$ to standard medium supplemented with $100 \mu \mathrm{M}$ ferrous perchlorate. A typical experiment is reported. Three other ones gave almost identical results.

at $200 \mu \mathrm{M}$ external concentration, takes up for about $50 \mathrm{nmol} / \mathrm{mg}$ prot of RLM in $30 \mathrm{~min}$ of incubation. The observation that the presence of the protonophore FCCP, which completely abolishes the electrochemical gradient, strongly prevents $\mathrm{Co}^{2+}$ uptake, suggests the involvement of an energy-dependent mechanism for this phenomenon. This observation is in disagreement with a previous report stating that $\mathrm{Co}^{2+}$ does not enter in mitochondria (Kroemer et al., 2007). Indeed, the addition of $200 \mu \mathrm{M} \mathrm{Co}^{2+}$ to the RLM suspension does induce a partial drop in $\Delta \Psi$ (Fig. 8, inset), suggesting that, at this concentration, the cation has a damaging effect on mitochondrial membrane which most probably reduces the rate and extent of this transport.

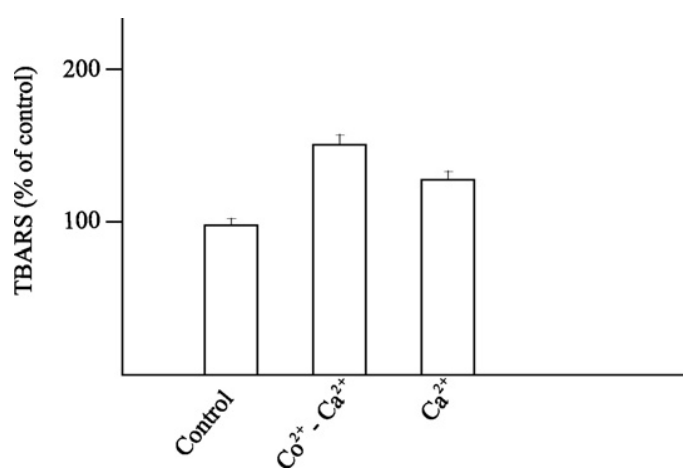

Fig. 7. Lipid peroxidation induced by $\mathrm{Co}^{2+}$ in the absence of $\mathrm{Ca}^{2+}$. Experimental conditions and cation concentrations as in Fig. 4. Data are expressed as percentage of TBARS generation and represent average \pm mean S.D. of six independent experiments. 


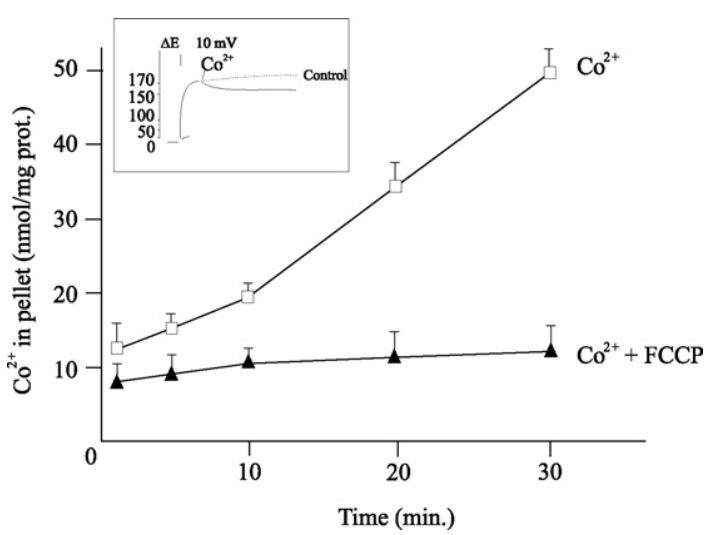

Fig. 8. Uptake of $\mathrm{Co}^{2+}$ by RLM. RLM were incubated in standard medium deprived of $\mathrm{Ca}^{2+}$, as described in Section 2. $\mathrm{Co}^{2+}$ was present at $200 \mu \mathrm{M}$ concentration. The inset shows the effect of $\mathrm{Co}^{2+}$ on $\Delta \psi$. When present, $1 \mu \mathrm{M}$ FCCP, $1 \mu \mathrm{M}$ RR, $1 \mathrm{mM}$ EGTA, $1 \mu \mathrm{M}$ CsA. Mean values \pm S.D. of four experiments.

(A)

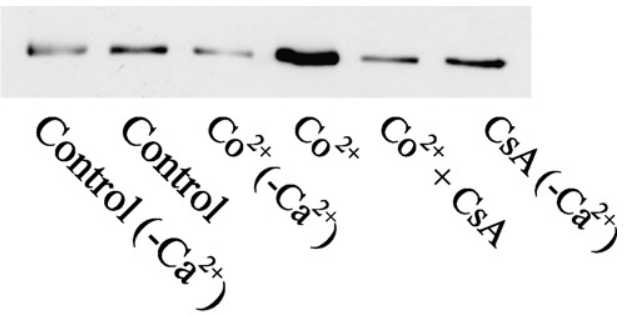

(B)

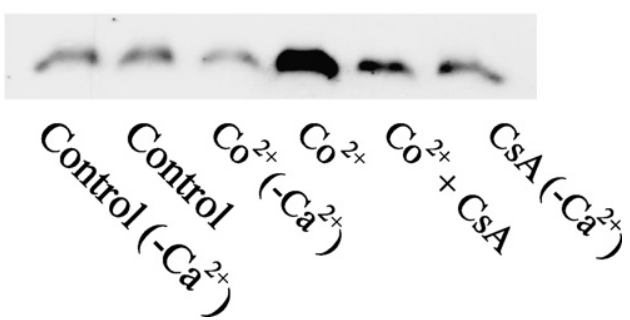

Fig. 9. Release of $\operatorname{AIF}(A)$ and cyt $c(B)$ from RLM induced by $\mathrm{Co}^{2+}$. Incubation conditions and $\mathrm{Co}^{2+}$ concentrations as in Fig. 4. Experiments replicated three times gave comparable results.

As the induction of the MPT may trigger the apoptotic pathway, the subsequent experiments evidence this possibility. As reported in the western blots of Fig. 9, the presence of $\mathrm{Co}^{2+}$ together $\mathrm{Ca}^{2+}$, which promotes the opening of the transition pore, induces the
(A)

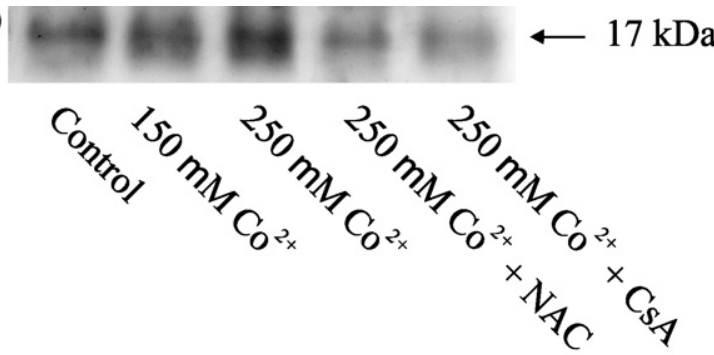

(B)

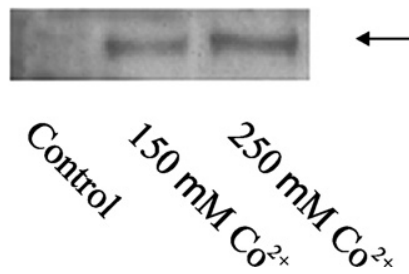

Fig. 11. Dose-dependent activation of caspase-3 (A) and HIF- $1 \alpha$ accumulation (B) by $\mathrm{Co}^{2+}$ in cultured hepatocytes. Hepatocytes were cultured in absence or presence of $\mathrm{Co}^{2+}$, at indicated concentrations. (A) 17-kDa band indicates major cleavage product of pro-caspase 3 . Where present, $5 \mathrm{mM} \mathrm{N}$-acetyl-cysteine (NAC) or $1 \mu \mathrm{M}$ cyclosporin $\mathrm{A}$ (CsA) were added and (B) $130-\mathrm{kDa}$ band indicates HIF-1 $\alpha$ protein.

release from mitochondria of AIF (panel A) and cyt c (panel B), two mitochondrial factors closely involved in apoptosis.

Taking into account all these observations on isolated RLM a subsequent aim of this study was to identify the effect of $\mathrm{CO}^{2+}$ at cellular level, in particular just on apoptosis induction. The results of Fig. 10 show that $\mathrm{Co}^{2+}$ induces cell death with apoptotic phenotype in rat hepatocyte primary cultures. The frequency of apoptosis, evaluated by visualizing nuclear shrinkage/fragmentation, with Hoechst dye 33258 staining, evidence a dose-dependent increase ( $\sim 4.5 \%$ with $150 \mu \mathrm{M} \mathrm{Co}^{2+}$ (not shown), 7.6\% with $250 \mu \mathrm{M} \mathrm{Co}^{2+}$ (panel B), $\sim 0.3 \%$ in untreated culture (panel A)). Upon apoptotic stimulus, caspase 3 , one of the main execution proteases of apoptosis, is activated, and generates one large subunit of $17 \mathrm{kDa}$ and a small one of $11 \mathrm{kDa}$. In cobalt-treated rat hepatocyte cultures, a dose-dependent increase in the $17 \mathrm{kDa}$ fragment is evidenced (Fig. 11A), whereas NAC or CsA can prevent caspase- 3 activation. As expected in our cell cultures, $\mathrm{Co}^{2+}$ also stabilizes transcription factor HIF-1, by inhibition of its degradation. The presence of increasing $\mathrm{Co}^{2+}$ concentrations (up to $250 \mu \mathrm{M}$ ), for $24 \mathrm{~h}$ induces proportional accumulation of this factor, as shown by western blot analysis (Fig. 11B). The different biological systems utilized in this study, isolated RLM or hepatocytes,
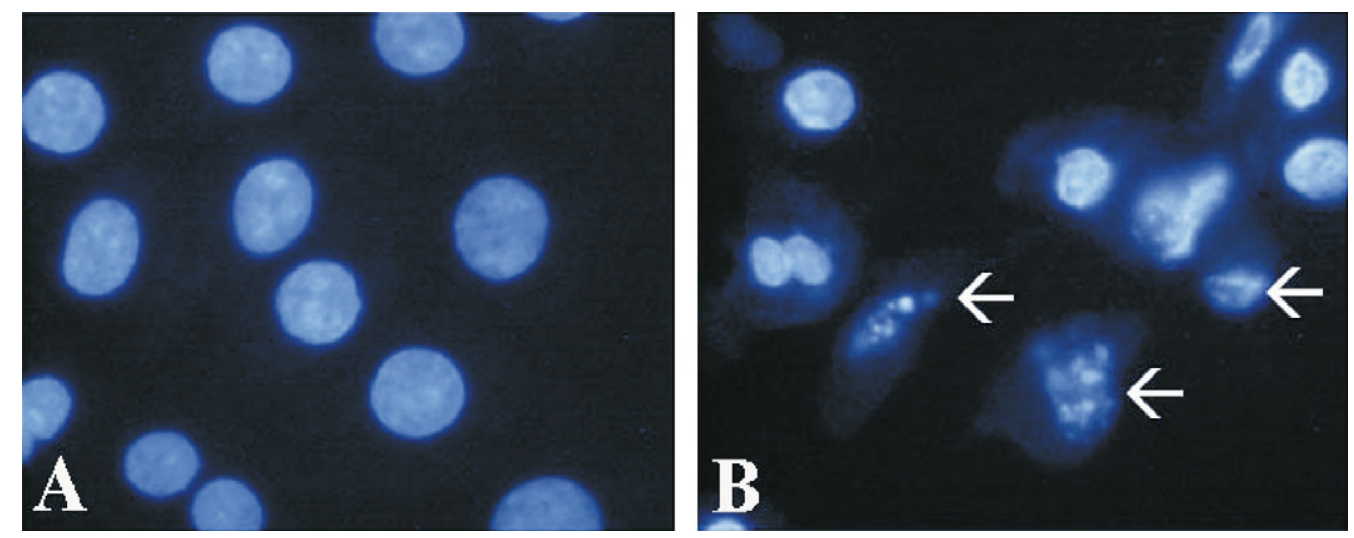

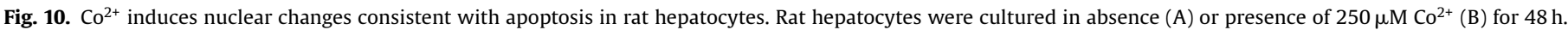
Cultures were then stained with Hoechst 33342 DNA-specific dye. Fragmented nuclei (arrows), indicative of apoptosis, were visualized by fluorescence microscopy. 
account for the different concentrations of $\mathrm{Co}^{2+}, 10$ or $250 \mu \mathrm{M}$, able to inducing its specific effects. It should be stressed that CsA, although inhibiting caspase-3 activation in hepatocytes (Fig. 11A), does not protect the cells against apoptosis but, by an unknown mechanism, further damages them in the presence of $\mathrm{Co}^{2+}$ (results not reported). This effect has also been observed by other authors (e.g., see Perez de Hornedo et al., 2007).

\section{Discussion}

The interaction of $\mathrm{Co}^{2+}$ with rat liver mitochondria, in the presence of $\mathrm{Ca}^{2+}$ result in the induction of MPT. Most probably, in order to provoke this effect, $\mathrm{Co}^{2+}$ has to enter the mitochondrial matrix by means of an energy-dependent mechanism as demonstrated by the results shown in Fig. 8.

Induction of the MPT by $\mathrm{Co}^{2+}$ is clearly demonstrated by the mitochondrial swelling observed in Figs. 1 and 2 and the parallel $\Delta \Psi$ collapse showed in Fig. 3. Complete or partial inhibition of both these events by the typical MPT inhibitor, CsA, and the $\mathrm{Ca}^{2+}$ transport inhibitors, RR and EGTA (Figs. 2 and 3A) clearly confirms the above statement.

The involvement of the AdNT in pore opening is demonstrated by the inhibition of mitochondrial swelling and $\Delta \Psi$ collapse exhibited by the AdNT ligands, ADP, ATP and BKA (Figs. 2 and 3B). Indeed the inhibition of the phenomenon by anti-oxidant $B H T$ and reductants DTE and NAC (Figs. 2 and 3C), demonstrates that oxidative stress is also involved in MPT induction. The slight effect of the bivalent cations, $\mathrm{Mg}^{2+}$ and $\mathrm{Mn}^{2+}$, well-known inhibitors of the phenomenon, suggests that $\mathrm{Co}^{2+}$ interacts at the level of the binding sites of these cations, thus preventing their effect (Figs. 2 and 3D).

The involvement of oxidative stress induced by $\mathrm{Co}^{2+}$, as a first step in the opening of the transition pore, is demonstrated by the results of Figs. 4 and 5, which show that $\mathrm{Co}^{2+}$ alone is able to oxidize sulfhydryl groups, glutathione and pyridine nucleotides, whereas oxidation is notably increased in the presence of $\mathrm{Ca}^{2+}$. Although thiol and glutathione oxidation in the absence of $\mathrm{Ca}^{2+}$ is negligible, only 4.5 or $6 \%$, respectively, (Fig. $4 \mathrm{~A}$ and B) it is statistically significant. In this regard, it should be emphasized that the oxidation of two critical cysteins located on AdNT (McStay et al., 2002), is sufficient to induce pore opening when $\mathrm{Ca}^{2+}$ is also present (for a review on MPT see Zoratti and Szabò, 1995). The oxidation of very low amounts of thiols resulting in an oxidative stress has also been observed in other investigations (e.g., see DallaVia et al., 2006). The extensive thiol, glutathione and pyridine nucleotide oxidation and $\mathrm{H}_{2} \mathrm{O}_{2}$ production in the presence of $\mathrm{Ca}^{2+}$ is the result of pore opening. In fact, in the presence of CsA, these effects are almost completely abolished. The residual oxidation and $\mathrm{H}_{2} \mathrm{O}_{2}$ formation are those due to $\mathrm{Co}^{2+}$ and $\mathrm{Ca}^{2+}$ but with the pore closed.

Besides opening the mitochondrial transition pore, the results of Fig. 3 indicate that $\mathrm{Co}^{2+}$ also induces some aspecific damage on mitochondrial inner membrane. As the figure shows, all the typical inhibitors of MPT, except RR and EGTA, exhibit only a partial protection on $\Delta \Psi$, suggesting that $\mathrm{Co}^{2+}$ causes proton leaks on the inner membrane. The complete protection exhibited by EGTA and RR is not completely attributable to their prevention on MPT. EGTA chelates $\mathrm{Co}^{2+}$, which blocks the uptake of the cation by RLM, whereas RR is ineffective (results not reported). Thus, the complete protection by EGTA is explained, the effect of RR remains obscure.

The possibility that $\mathrm{Co}^{2+}$ can induce oxidative stress in hepatocytes by increasing the formation of ROS, with the results of provoking cell death has been examined also by other authors (Pourahmad et al., 2003).

Our proposed mechanism of ROS production by $\mathrm{Co}^{2+}$ in liver cells takes into account a previous demonstration regarding ROS produc- tion by $\mathrm{Ca}^{2+}$ in liver mitochondria (Grijalba et al., 1999) following $\mathrm{Ca}^{2+}$ interactions with membrane cardiolipins. Most probably $\mathrm{Co}^{2+}$, as proposed for $\mathrm{Ca}^{2+}$, induces tight cardiolipin packing, resulting in a molecular rearrangement of the membrane. This leads to an alteration in ubiquinone mobility which favors increased production of the semiquinone radical. Subsequently, $\mathrm{Co}^{2+}$ alone interacts with molecular oxygen by forming superoxide anion, $\mathrm{O}_{2}{ }^{\bullet-}$, which, by a dismutation reaction, catalyzed by superoxide dismutase, produces an increase in hydrogen peroxide when compared with the condition without $\mathrm{Ca}^{2+}$ (Fig. 6A).

The results of Fig. 6A also confirmation the above proposal, showing that $\mathrm{Ca}^{2+}$ alone (see control) also increases $\mathrm{H}_{2} \mathrm{O}_{2}$ production.

The further increase in $\mathrm{H}_{2} \mathrm{O}_{2}$ generation observed in the presence of both cations (Fig. 6A) is due to augmented oxidative stress as a result of pore opening.

$\mathrm{H}_{2} \mathrm{O}_{2}$, separately produced by $\mathrm{Co}^{2+}$ and $\mathrm{Ca}^{2+}$, interacts with the $\mathrm{Fe}^{2+}$ of $\mathrm{Fe}-\mathrm{S}$ centers belonging to the iron sulfur proteins of the respiratory chain, by means of a Fenton or Haber-Weiss reaction, leading to generation of the highly damaging hydroxyl radical. This species is most probably responsible for the observed oxidative stress. The increase in lipid peroxidation by both cations strongly supports this proposal (Fig. 7). However a clear confirm of this suggestion is given by the generation of highly ROS observed in Fig. 6B. Taking into account that the probe APF exhibits fluorescence augmentation only upon reaction with ${ }^{-} \mathrm{OH}, \mathrm{ONOO}^{-}$and ${ }^{-} \mathrm{OCl}$ but not with $\mathrm{O}_{2}{ }^{\bullet-}, \mathrm{H}_{2} \mathrm{O}_{2},{ }^{1} \mathrm{O}_{2}$, NO, $\mathrm{ROO} \bullet$ (Setsukinai et al., 2003), in consideration of the above-mentioned reactions, it is possible to state that the highly damaging ROS responsible for the observed oxidative stress is the hydroxyl radical.

These results, however, raise a question. Why, although $\mathrm{Ca}^{2+}$ induces oxidative stress, does it not open the pore? One explanation is that hydroxyl radical generation is less than that with $\mathrm{Co}^{2+}$ (see Fig. 7). Alternatively, ROS generation by $\mathrm{Ca}^{2+}$ may take place away from the critical cysteins, located on AdNT, whose oxidation is responsible for pore opening (McStay et al., 2002).

The opening of the transition pore accounts for the release of pro-apoptotic factors, AIF, and cyt $c$, demonstrated by the results of Fig. 9 and suggesting the possibility of the intrinsic apoptotic pathway triggering when the phenomenon takes place in a cell system. This is clearly confirmed by the results reported in Fig. 10, showing that $\mathrm{Co}^{2+}$ induces apoptosis in rat hepatocyte primary cultures. This demonstrates that all the events linked to $\mathrm{Co}^{2+}$ action, observed at mitochondrial level, and having the result of opening the transition pore, are very close to the induction of the intrinsic apoptosis in hepatocyte cultures. At molecular level, apoptosis event is characterized by the activation of several cysteine proteases, called caspases. Upon the apoptotic stimulus, initiator caspases can in turn cleave specific protein substrates resulting in the apoptotic phenotype (Nicholson, 1999). As shown in Fig. 11A, caspase 3, normally synthesized as an inactive pro-enzyme is activated by $\mathrm{Co}^{2+}$ to generate the $17 \mathrm{kDa}$ subunit, thus demonstrating triggering of the pro-apoptotic pathway (Hengartner, 2000). The observation that NAC and CsA prevent caspase- 3 activation supports the proposal that apoptosis induction is due to an oxidative stress and depends on the opening of the mitochondrial transition pore.

A large number of studies (see Semenza, 2001) have reported that $\mathrm{Co}^{2+}$ mimics hypoxia by stabilizing HIF- $1 \alpha$, by inhibition of its degradation. HIF- $1 \alpha$ is synthesized continuously, and hydroxylation of at least one of two critical proline residues in specific domain promotes its interaction with the von Hippel-Lindau (VHL) E3 ubiquitin ligase complex which targets it for rapid proteasomal degradation under normoxic conditions (Ivan et al., 2001). Hydroxylation is catalyzed by HIF prolyl 4-hydroxylases (2-oxoglutarate dioxygenases, requiring $\mathrm{Fe}^{2+}, 2$-oxoglutarate, $\mathrm{O}_{2}$, and ascorbate). 
Hydroxylation of a specific asparagine residue in the C-terminal transactivation domain of HIF- $1 \alpha$ prevents its interaction with the transcriptional coactivator p300 (Lando et al., 2002). The asparaginyl hydroxylase belongs to the 2-oxoglutarate dioxygenase and requires the same cosubstrates as the prolyl 4-hydroxylases. It has been suggested that cobalt stabilizes HIF by inhibiting prolyl and/or asparaginyl-hydroxylases through many mechanisms: occupying their $\mathrm{Fe}^{2+}$ binding site, depleting intracellular ascorbate levels favouring iron oxidation or binding to HIF preventing interaction with VHL.

The observation that $\mathrm{Co}^{2+}$ increases the level of HIF- $1 \alpha$ (Fig. 11B) further confirms the induction of apoptosis by $\mathrm{Co}^{2+}$. HIF- $1 \alpha$ levels have also been found to increase also during brain ischemia in the rat (Jones and Bergeron, 2001; Wiener et al., 1996) and is associated with markers of apoptosis (Yu et al., 2001).

In conclusion, we provide much evidence that cobalt causes intrinsic apoptosis in hepatocytes primary cultures by activation of caspase 3 and stabilization with injured level of HIF-1 $\alpha$. This is due to mitochondrial damage by oxidative stress induced by $\mathrm{Co}^{2+}$, with release of apoptotic factors. Indeed, this study also shows that $\mathrm{Co}^{2+}$ has damaging effects on mitochondrial inner membrane, thus affecting its proton impermeability (see Fig. 3). It should also be noted that, apparently, the results reported here seem to be in contrast with a previous paper stating that the targets of $\mathrm{Co}^{2+}$ to produce ROS are lysosomes instead of mitochondria, as ATP glycolytic generators cannot prevent oxidative stress in hepatocytes (Pourahmad et al., 2003). The results reported here do not exclude the participation of lysosomes in oxidative stress leading to apoptosis, but the involvement of mitochondria is unequivocally proven. The discrepancy may be explained by taking into account the fact that $\mathrm{Co}^{2+}$, as mentioned above, even at $10 \mu \mathrm{M}$ concentration, partially affects membrane impermeability to protons (Fig. 3). In addition, preliminary analyses of cell morphology versus cell permeability by flow fluorocytometry indicate that $200 \mu \mathrm{M} \mathrm{Co}^{2+}$ not only induces apoptosis, but is also able to induce necrosis in a low percentage of hepatocytes (results not reported). These observations highlight the importance of $\mathrm{Co}^{2+}$ concentration in inducing apoptosis and/or necrosis. Most probably, at the $500 \mu \mathrm{M}$ concentration used in the quoted study (Pourahmad et al., 2003), $\mathrm{Co}^{2+}$ induces necrotic damage, which can no longer be restored by ATP generators, explaining the apparent disagreement between the results reported here and those quoted above (Pourahmad et al., 2003).

\section{Acknowledgement}

We thank Mario Mancon who was involved in technical support of this work.

\section{References}

Araya J, Maruyama M, Inoue A, Fujita T, Kawahara J, Sassa K, et al. Inhibition of proteasome activity is involved in cobalt-induced apoptosis of human alveolar macrophages. Am J Physiol Lung Cell Mol Physiol 2002;283:849-58.

Blazyk JF, Steim JM. Phase transition in mammalian membranes. Biochim Biophys Acta 1972;266:737-41.

Bragadin M, Toninello A, Mancon M, Manente S. The interactions of cobalt(II) with mitochondria from rat liver. J Biol Inorg Chem 2007;12:631-5.

Bruick RK. Expression of the gene encoding the pro-apoptotic Nip3 protein is induced by hypoxia. Proc Natl Acad Sci USA 2000;97:9082-7.

Chandel NS, Vander Heiden MG, Thompson CB, Schumacker PT. Redox regulation of p53 during hypoxia. Oncogene 2000;19:3840-8.

DallaVia L, Marini AM, Salerno S, Toninello A. Mitochondrial permeability transition induced by novel pyridothiopyranopyrimidine derivatives: potential new antimitochondrial antitumour agents. Biochem Pharmacol 2006;72:1657-67.

Derelank MJ, Hollinger MA. Handbook on the toxicology of metals. 2nd edition. CRC Press; 2002. p. 924.

Epstein AC, Gleadle JM, McNeill LA, Hewitson KS, O'Rourke J, Mole DR, et al. C. elegans EGL-9 and mammalian homologs define a family of dioxygenases that regulate HIF by prolyl hydroxylation. Cell 2001;107:43-54.
Gardini G, Cabella C, Cravanzola C, Vargiu C, Belliardo S, Testore G, et al. Agmatine induces apoptosis in rat hepatocyte cultures. J Hepatol 2001;35:482-9.

Gornall AG, Bardawill CJ, David MM. Determination of serum proteins by means of the biuret method. J Biol Chem 1949;177:751-66.

Grijalba MT, Vercesi AE, Schreier S. $\mathrm{Ca}^{2+}$-induced increased lipid packing and domain formation in submitochondrial particles. A possible early step in the mechanism of $\mathrm{Ca}^{2+}$ stimulated generation of reactive oxygen species by the respiratory chain. Biochemistry 1999;38:13279-87.

Hengartner MO. The biochemistry of apoptosis. Nature 2000;407:770-6.

Huk OL, Catelas I, Mwale F, Antoniou J, Zukor DJ, Petit A. Induction of apoptosis and necrosis by metal ions in vitro. J Arthroplasty 2004;9:84-7.

Ivan M, Kondo K, Yang H, Kim W, Valiando J, Ohh M, et al. HIF- $\alpha$ targeted for VHLmediated destruction by proline hydroxylation: implications for $\mathrm{O}_{2}$ sensing. Science 2001;292:464-8.

Jensen BD, Gunter KK, Gunter TE. The efficiences of the component steps of oxidative phosphorylation. II. Experimental determination of the efficiencies in mitochondria and examination of the equivalence of membrane potential and pH gradient in phosphorylation. Arch Biochem Biophys 1986;248: 305-23.

Jones NM, Bergeron M. Hypoxic preconditioning induces changes in HIF-1 target genes in neonatal rat brain. J Cereb Blood Flow Metab 2001;21:1105-14.

Kamo N, Muratsugu M, Hongoh R, Kobatake Y. Membrane potential of mitochondria measured with an electrode sensitive to tetraphenyl phosphonium and relationship between proton electrochemical potential and phosphorylation potential in steady state. J Membr Biol 1979;49:105-21.

Karovic O, Tonazzini I, Rebola N, Edstrom E, Lovdahl C, Fredholm BB, et al. Toxic effects of cobalt in primary cultures of mouse astrocytes. Similarities with hypoxia and role of HIF-1 alpha. Biochem Pharmacol 2006;73:694-708.

Kroemer G, Galluzzi L, Brenner C. Mitochondrial membrane permeabilization in cell death. Physiol Rev 2007;87:99-163.

Lando D, Peet DJ, Whelan DA, Gorman JJ, Whitelaw ML. Asparagine hydroxylation of the HIF transactivation domain-a hypoxic switch. Science 2002;295: 858-61.

Loschen G, Azzi A, Flohè L. Mitochondrial $\mathrm{H}_{2} \mathrm{O}_{2}$ formation at site II. FEBS Lett 1973;33:84-7.

McStay GP, Clarke SJ, Halestrap AP. Role of critical thiol groups on the matrix surface of the adenine nucleotide traslocase in the mechanism of the mitochondrial permeability transition pore. Biochem J 2002;367:541-8.

Morwenna RK, Ratcliffe WPJ. Mammalian oxygen sensing and hypoxia-inducible factor-1. Int J Biochem Cell Biol 1997;29:1419-32.

Nicholson DW. Caspase structure, proteolytic substrates, and function during apoptotic cell death. Cell Death Differ 1999;6:1028-42.

Norusis MJ. SPSS for Windows Base System User's Guide Release 6.0. Chicago: SPSS; 1993.

Olivieri G, Hess C, Savaskan E, Ly C, Meier F, Baysang G, et al. Muller-Spahn F, Melatonin protects SHSY5Y neuroblastoma cells from cobalt-induced oxidative stress, neurotoxicity and increased beta-amyloid secretion. J Pineal Res 2001;31:320-5.

Perez de Hornedo J, de Arriba G, Calvino-Fernandez M, Benito S, Parra Cid T. Cyclosporin A causes oxidative stress and mitochondrial dysfunction in tubular renal cells. Nefrologia 2007;27:565-73.

Pourahmad J, O'Brien PJ, Jokar F, Daraei B. Carcinogenic metal induced sites of reactive oxygen species formation in hepatocytes. Toxicol In Vitro 2003;17: 803-10.

Probst I, Unthan-Fechner K. Activation of glycolysis by insulin with a sequential increase of the 6-phosphofructo-2 kinase activity, fructose 2,6-bisphosphate level and pyruvate kinase activity in cultured rat hepatocytes. Eur J Biochem 1982;153:347-53.

Pulido MD, Parrish AR. Metal-induced apoptosis: mechanisms. Mutat Res 2003;533:227-41.

Reznick Z, Packer L. Oxidative damage to proteins: spectrophometric method for carbonyl essay. Methods Enzymol 1994;233:357-63.

Ricchelli F, Gobbo S, Moreno G, Salet C. Changes of the fluidity of mitochondrial membranes induced by the permeability transition. Biochemistry 1999;38:9295-300.

Santos AC, Uyemura SA, Lopes JL, Bazon JN, Mingatto FE, Curti C. Effect of naturally occurring flavonoids on lipid peroxidation and membrane permeability transition in mitochondria. Free Radic Biol Med 1998;24:1455-61.

Schneider WC, Hogeboom GH. Intracellular distribution of enzymes. V. Further studies on the distribution of cytochrome $c$ in rat liver homogenate. J Biol Chem 1950;183:123-8.

Semenza GL. HIF-1, $\mathrm{O}_{2}$, and the 3 PHDs: how animal cells signal hypoxia to the nucleus? Cell 2001;107:1-3.

Setsukinai K-I, Urano Y, Kakinuma K, Majima HJ, Nagano T. Development of novel fluorescence probes that can reliably detect reactive oxygen species and distinguish specific species. J Biol Chem 2003;31:3170-5.

Tietze F. Enzymic method for quantitative determination of nanogram amounts of total and oxidized glutathione: applications to mammalian blood and other tissues. Anal Biochem 1969;27:502-22.

Tomaro ML, Frydman J, Frydman RB. Heme oxygenase induction by $\mathrm{CoCl}_{2}, \mathrm{Co}-$ protoporphyrin IX, phenylhydrazine, and diamide: evidence for oxidative stress involvement. Arch Biochem Biophys 1991;286:610-7.

Toninello A, Di Lisa F, Siliprandi D, Siliprandi N. Uptake of spermine by rat liver mitochondria and its influence on the transport of phosphate. Biochim Biophys Acta 1985;815:399-404. 
Wang G, Harra TK, Mitra S, Lee HM, Englander EW. Mitochondrial DNA damage and a hypoxic response are induced by $\mathrm{Cocl}_{2}$ in rat neuronal PC12 cells. Nucleic Acids Res 2000;28:2135-40.

Wiener CM, Booth G, Semenza GL. In vivo expression of mRNAS encoding hypoxiainducible factor 1. Biochem Biophys Res Commun 1996;225:485-8.

Willis ED, Wilkinson AE. Release of enzymes from lysosomes by irradiation and the relation of lipid peroxide formation to enzyme release. Biochem J 1966;99:657-64.

Yang SJ, Pyen J, Lee I, Lee H, Kim Y, Kim T. Cobalt chloride-induced apoptosis and extracellular signal-regulated protein kinase $1 / 2$ activation in rat C6 glioma cells. J Biochem Mol Biol 2004;37:480-6.
Yu R, Geo L, Jiang S, Guan P, Mao B. Association of HIF-1 $\alpha$ expression and cell apoptosis after traumatic brain injury in the rat. Chin J Traumatol 2001;4 218-21.

Zoratti M, Szabò I. The mitochondrial permeability transition. Biochem Biophys Acta 1995;1241:139-76.

Zou W, Yan M, Xu W, Huo H, Sun L, Zheng Z, et al. Cobalt chloride induces PC12 cells apoptosis through reactive oxygen species and accompanied by AP-1 activation. J Neurosci Res 2001;64:646-53.

Zou W, Zeng J, Zhuo M, Xu W, Sun L, Wang J, et al. Involvement of caspase-3 and p38 mitogen-activated protein kinase in cobalt chloride-induced apoptosis in PC12 cells. J Neurosci Res 2002;67:837-43. 